Documentation et bibliothèques

DOCUMENTATION BIBLIOTHEQUES

\title{
L'évolution de la coopération dans les bibliothèques de collège du Québec
}

\section{Jean-M. Bernard Léveillé}

Volume 23, numéro 3, septembre 1977

URI : https://id.erudit.org/iderudit/1055226ar

DOI : https://doi.org/10.7202/1055226ar

Aller au sommaire du numéro

Éditeur(s)

Association pour l'avancement des sciences et des techniques de la documentation (ASTED)

ISSN

0315-2340 (imprimé)

2291-8949 (numérique)

Découvrir la revue

Citer cet article

Léveillé, J.-M. B. (1977). L'évolution de la coopération dans les bibliothèques de collège du Québec. Documentation et bibliothèques, 23(3), 135-141.

https://doi.org/10.7202/1055226ar
Résumé de l'article

La présentation des divers travaux de coopération réalisés dans le domaine des bibliothèques de collège au Québec depuis quelques décennies, particulièrement depuis le début des années soixante, montre que la coopération a été vivante dans ce genre de bibliothèques.
Tous droits réservés (c) Association pour l'avancement des sciences et des techniques de la documentation (ASTED), 1977
Ce document est protégé par la loi sur le droit d'auteur. L'utilisation des services d'Érudit (y compris la reproduction) est assujettie à sa politique d'utilisation que vous pouvez consulter en ligne.

https://apropos.erudit.org/fr/usagers/politique-dutilisation/ 


\title{
L'évolution de la coopération dans les bibliothèques de collège du Québec
}

\author{
Jean-M. Bernard Léveillé \\ Service des bibliothèques d'enseignement \\ Service général des moyens d'enseignement \\ Montréal
}

La présentation des divers travaux de coopération réalisés dans le domaine des bibliothèques de collège au Québec depuis quelques décennies, particulièrement depuis le début des années soixante, montre que la coopération a été vivante dans ce genre de bibliothèques.

This presentation of different cooperative projects undertaken in college libraries in Québec in the last few decades, especially since the beginning of the 60's, shows that cooperation has been quite lively in this type of library.

La presentacion de diversos trabajos de cooperacion realizados en el dominio de las bibliotecas de colegio de Quebec desde algunos decenios y particularmente desde al principio de los anos 60 demuestra que la cooperacion fue viva en este tipo de bibliotecas.

Notre intention est de dresser un bilan des travaux réalisés au profit des bibliothèques de collège, grâce à des efforts concertés et collectifs. Malgré toutes les subtilités linguistiques, nous utiliserons à l'occasion les mots "coopération", "collaboration", "participation» et "concertation» pour souligner des réalisations qui n'auraient jamais été possibles sans cette volonté des bibliothécaires de collège de travailler en équipe et de mettre en commun des ressources intellectuelles et, parfois, des ressources financières.

\section{Trois grandes étapes dans la vie des bibliothèques de collège}

De la création des premiers collèges classiques jusqu'au milieu des années quarante
Un réel souci d'acquérir de la documentation et de veiller à sa conservation marque cette période. II faut considérer qu'à cette époque, il y avait peu de spécialistes dans le domaine de l'organisation scientifique des bibliothèques. De plus, les programmes d'étude étaient peu axés sur I'utilisation des bibliothèques. Le Québec, par ailleurs, ne possédait pas encore de bibliothèque nationale. Plusieurs collèges ont eu la clairvoyance de jouer en quelque sorte un rôle de suppléance en constituant des collections dont une partie avait une valeur certaine, particulièrement au niveau des canadiana et des laurentiana. Parmi les pionniers des collèges québécois, il y avait des éducateurs chevronnés dont plusieurs, venus de France, connaissaient bien la littérature en général. Avec des moyens financiers pitoyables, ils ont permis au Québec de conserver des biens culturels 
qui, autrement, auraient pu facilement franchir les frontières.

\section{Du milieu des années quarante jusqu'à 1967}

La formation des premières générations de bibliothécaires à l'École de bibliothécaires de l'Université de Montréal, entre 1937 et 1962, puis à l'École de bibliothéconomie de la même institution par la suite, a alors commencé à porter des fruits au niveau de l'organisation des bibliothèques de collège. Peu après 1945, quelques collèges entreprirent la réorganisation de leur bibliothèque. À partir de 1950, ce mouvement s'accentua pour s'étendre à tout le réseau, principalement au cours des années soixante.

Cette période a été marquée par la réalisation de projets importants, grâce au leadership de quelques bibliothécaires qui ont contribué à valoriser la bibliothèque dans le milieu collégial. Nous reviendrons sur ce sujet de façon plus systématique. Pour l'instant, nous n'hésitons pas à affirmer que ces années de labeur ont été vécues avec un réel enthousiasme.

\section{De 1967 à nos jours}

L'implantation des collèges d'enseignement général et professionnel (cégeps) a débuté en 1967. Douze cégeps étaient créés dès cette année-là, treize en 1968, huit en 1969, cinq en 1970, quatre en 1971 et trois en 1972.

L'accroissement aussi rapide du réseau des collèges publics a posé de nombreux problèmes résultant du regroupement de plusieurs institutions, de l'augmentation considérable de la clientèle étudiante et de l'instauration de programmes d'étude comportant un grand nombre de disciplines nouvelles. Les collections des bibliothèques étaient nettement déficientes et il y avait pénurie de locaux pour accueillir des clientèles allant de 2,000 à 4,000 étudiants. II fallait aussi structurer les services de chaque bibliothèque en fonction des situations nouvelles.
Pour aider à solutionner la déficience des collections, la Direction générale de l'enseignement collégial (DGEC) a octroyé, à partir de 1969, des budgets d'immobilisation pour fonds de bibliothèque: $\$ 870$, 000 en 1969-1970; $\$ 1,081,400$ en $1970-1971 ; \$ 2,109,000$ en $1971-1972 ; \$ 1$, 856,000 en 1972-1973; $\$ 1,175,000$ en 1973-1974; $\$ 1,350,000$ en 1974-1975; $\$ 1$, 125,000 en 1975-1976; $\$ 835,000$ en 1976-1977; $\$ 800,000$ en 1977-1978.

\section{Organismes impliqués dans les travaux et les réalisations des bibliothèques de collège}

Lorsque nous aborderons le bilan proprement dit des résultats obtenus dans le milieu des bibliothèques de collège grâce à la concertation, il faudra y associer notamment la Direction générale de l'enseignement collégial (DGEC), la Fédération des collèges classiques (FCC), la Fédération des cégeps, l'Association des collèges privés (ACQ), l'Association canadienne des bibliothécaires de langue française (ACBLF), l'Association pour l'avancement des sciences et des techniques de la documentation (ASTED), l'Association des bibliothécaires du Québec (ABQ), le Service des bibliothèques d'enseignement et la Centrale des bibliothèques.

II ne serait pas inutile, croyons-nous, de souligner au passage que la FCC, autrefois, comme maintenant la Fédération des cégeps et I'ACQ, sont des organismes coopératifs où la participation financière de chaque institution membre rend possible l'action de nombreuses commissions (dont celle des directeurs de bibliothèque), ainsi que des sous-commissions, des groupes de travail, des comités spéciaux et permanents qui sont appuyés dans leurs travaux par les ressources du Centre d'animation, de développement et de recherche en éducation (CADRE).

\section{Les bibliothèques de collège et le bilan de leurs réalisations}

Nous avons déjà esquissé trois principales étapes dans la vie des bibliothèques 
de collège. Les actions les plus marquantes que nous allons retracer se situeront à partir de 1960 jusqu'à maintenant.

\section{Programme pour une bibliothèque collégiale}

En 1961, l'ACBLF publiait un ouvrage d'Edmond Desrochers intitulé Programme pour une bibliothèque collégiale. Avant de publier ce texte, l'auteur, malgré ses recherches importantes et sa très grande compétence, avait pris la précaution de le soumettre à plusieurs directeurs des études et à un comité de bibliothécaires professionnels, afin d'en garantir la pertinence tant au point de vue pédagogique que bibliothéconomique.

Cet esprit de concertation, de consultation et de collaboration, nous le retrouverons dans toutes les autres publications touchant les bibliothèques de collège. Nous signalons en premier lieu cette publication parce qu'elle visait à sensibiliser les administrateurs de collège à l'importance qu'il y avait de pouvoir compter sur une bibliothèque de qualité pour appuyer l'action pédagogique.

\section{Création d'une section des bibliothèques de collège au sein de l'ACBLF en 1961}

Cette section a organisé de nombreuses sessions d'étude sur divers sujets, comme en témoignent les comptes rendus publiés dans les Actes des congrès et dans les autres publications périodiques de l'ACBLF et de l'ASTED.

\section{Sessions d'étude du Lac Trèfle et les suites qui en ont découlé pour les bibliothèques de collège}

Au cours des étés 1961 et 1962, la faculté des Arts de l'Université de Montréal organisa des sessions intensives au Lac Trèfle, situé dans un décor enchanteur au nord du comté de Joliette. Les collèges y déléguèrent des directeurs d'étude, des professeurs et des bibliothécaires. Dans une atmosphère détendue, les délibéra- tions, fort sérieuses d'ailleurs, portèrent principalement sur l'évolution de la pédagogie vers l'école active. Cette réflexion en groupe précédait l'enquête de la Commission royale sur l'enseignement au Québec et la publication de son rapport en 1963.

Les sessions du Lac Trèfle permirent à un groupe de bibliothécaires de discuter librement de pédagogie avec les administrateurs des programmes d'étude et avec les enseignants.

Parmi les suites marquantes de ces sessions, on peut souligner:

- Une revalorisation de la bibliothèque dans le milieu collégial. On voulait que la pédagogie nouvelle fasse une plus grande place au travail personnel de l'étudiant; pour l'appuyer dans ses recherches, il apparut que la bibliothèque devenait un instrument essentiel. II fallait songer à organiser des centres de documentation valables et trouver des personnes qualifiées pour mener cette tâche à bonne fin. Plusieurs collèges décidèrent d'envoyer un des membres de leur personnel poursuivre des études en bibliothéconomie. Certains allèrent étudier à Montréal, d'autres à Washington. De préférence, ils devaient choisir pour leur thèse de maîtrise en bibliothéconomie des sujets susceptibles de servir toute la collectivité collégiale. Dans une collection portant le titre de Livres et bibliothèques, la FCC a publié au moins quatre thèses dont on trouvera la liste dans les sources regroupées à la fin de ce texte.

- Des cours d'initiation au travail en bibliothèque devinrent obligatoires pour tous les étudiants des classes avancées qui avaient à poursuivre des travaux de recherche. Le nombre d'heures du cours d'initiation variait entre trois et cinq heures. En 1964, le Bulletin de la F.C.C. consacrait entièrement son numéro 5 aux bibliothèques de collège.

- La création d'une Centrale de catalogage. Le raisonnement des bibliothécaires de collège pour promouvoir la création d'un tel organisme était très simple. Au moment où il fallait se lancer dans une opération géné- 
ralisée d'organisation des bibliothèques, il paraissait indéfendable qu'on multiplie les mêmes opérations catalographiques dans possiblement 97 collèges. Le 14 octobre 1964, l'assemblée générale de la FCC approuvait la création de la Centrale de catalogage. Chaque collège membre de la FCC a dû verser une cotisation pour permettre à cette Centrale de naître.

C'est un véritable esprit de coopération qui a présidé à la fondation de la Centrale de catalogage. Au cours des premières années, son directeur, Paul Francœur, a pu compter sur l'étroite collaboration de la Sous-commission des directeurs de bibliothèque de la FCC pour orienter ses actions. En plus de la mise sur pied des services catalographiques, il y eut aussi des efforts considérables visant à normaliser les formulaires dans les bibliothèques de collège. On fit même l'achat massif de fiches de catalogue dont les bibliothèques avaient besoin. En peu de temps, un million de fiches avaient été distribuées dans le réseau.

A ses débuts, la Centrale de catalogage devait servir le milieu collégial. Peu à peu, elle a étendu ses services aux bibliothèques scolaires. Aujourd'hui, elle travaille aussi pour les bibliothèques publiques.

En 1966, la Centrale de catalogage est devenue la Centrale des bibliothèques. II n'est pas possible d'élaborer longuement ici autour des nombreuses publications qu'elle a mises au service des bibliothèques. Nous croyons qu'elle a contribué de façon importante à l'organisation des bibliothèques malgré toutes les critiques qui ont pu lui être adressées périodiquement. S'il fallait, en 1977, que chaque bibliothèque règle isolément tous ses problèmes de catalogage, construise ses propres instruments bibliographiques et dépouille par ses propres moyens ses collections de périodiques, jusqu'où pourrait aller l'escalade des budgets?

Face à l'urgence de développer des collections valables pour les cégeps et à la nécessité d'utiliser à bon escient les bud- gets de rattrapage mis à leur disposition à cette fin, les directeurs de bibliothèque de cégep acceptèrent, le 31 mars 1971, de verser provisoirement une cotisation à la Centrale des bibliothèques, dans le but de lui permettre de développer un Centre de bibliographie à l'intention des collèges. Cette cotisation est encore en vigueur en 1977. Elle représente un montant de $\$ 270,000$ qui permet aux cégeps d'utiliser, lorsque possible, les services bibliographiques et catalographiques de la Centrale, ainsi que son service de dépouillement des périodiques.

Un comité de liaison entre la Centrale des bibliothèques et les cégeps a été créé en 1971. II a pour mandat de veiller à ce que les services de la Centrale correspondent aux besoins des bibliothèques de collège. Ce comité a tenu trente réunions entre le 3 juin 1971 et le 25 mai 1977.

\section{Normes de construction des bibliothèques de collège}

En 1967, au moment où démarrait l'implantation du réseau des cégeps, on constata qu'il faudrait entreprendre un programme de construction de bibliothèques. La DGEC manifesta, dès ce moment, le désir de pouvoir s'appuyer sur des normes pour étudier les projets de construction qui lui seraient éventuellement soumis, pour les approuver ainsi que pour attribuer les fonds nécessaires à leur réalisation. On forma un comité "ad hoc» composé de trois délégués de la DGEC, du directeur du Service des bibliothèques d'enseignement et de cinq directeurs de bibliothèque de collège. Après une année de travail du comité, la DGEC publia, en 1968, un Guide pour la construction des bibliothèques de cégeps. La DGEC a su utiliser ce Guide avec profit à l'occasion des nombreux projets de construction de bibliothèque qui lui ont été soumis.

En 1968, nous avions une connaissance assez rudimentaire du développement éventuel des audiovidéothèques. Cette partie du Guide comportait des faiblesses. Des programmes de construction élaborés dans les années qui suivirent ainsi que des 
rapports sur l'audiovidéothèque, publiés par deux comités, en 1970 et en 1973, ont contribué à combler certaines lacunes.

En 1975, vingt-quatre bibliothèques de cégep avaient été construites et six autres étaient en chantier. Aujourd'hui, l'aménagement des bibliothèques dans le réseau des cégeps est sur le point d'être complété. Les bibliothécaires de collège ont collaboré à l'élaboration de leurs propres normes de construction, ils se sont entraidés à l'occasion de la préparation des programmes de construction et de l'étude des plans préparés par des architectes.

\section{Normes d'excellence des bibliothèques de collège}

Face aux nouvelles orientations pédagogiques des collèges, les directeurs de bibliothèque décidèrent en 1972 d'élaborer des normes d'excellence. Pas moins de quinze personnes travaillèrent, durant une année, à rédiger un texte préliminaire qui fut soumis et approuvé par l'ensemble des coordonnateurs de bibliothèque à l'occasion de leur assemblée annuelle des 29 et 30 novembre 1973. Le texte final fut publié en 1974 et présenté aux directeurs généraux qui demandèrent de quantifier ces normes. Un autre groupe de travail se mit à la tâche et on publia un deuxième document en 1975.

Malheureusement, les normes dans leur ensemble ont été mises en veilleuse en raison des difficultés budgétaires que connaissent présentement les collèges. Elles n'ont donc pas reçu une reconnaissance officielle. Cependant, nous savons qu'elles sont connues et même utilisées à titre de référence à certains paliers du ministère de l'Éducation.

\section{Critères pour le choix de la documentation des bibliothèques de collège}

Un premier comité "ad hoc» avait établi des critères généraux de choix des documents pour toutes les bibliothèques d'enseignement, au cours de l'année
1969-1970. Un second comité, formé au mois de septembre 1975, reçut comme mandat de fixer des critères généraux touchant le choix de la documentation qui convient aux collèges. Huit personnes participèrent aux travaux de ce comité. Leur rapport, publié le 31 mars 1975, a été largement diffusé dans tout le réseau collégial.

\section{Constitution d'un dossier statistique des bibliothèques de collège depuis 1968}

Les directeurs de bibliothèque ont reconnu, d'une façon générale, qu'il est normal qu'une saine administration possède des chiffres relativement à sa clientèle, à ses ressources humaines, matérielles et financières, relativement aussi à l'utilisation des ressources disponibles. Grâce à la collaboration des collèges, le Service des bibliothèques d'enseignement a réussi à constituer, depuis 1968, un dossier statistique qui s'est amélioré d'année en année.

Les relevés statistiques pour l'ensemble des bibliothèques du réseau collégial - et c'est là le plus important - sont de plus en plus diffusés et utilisés.

\section{Les bibliothécaires de collège face aux préoccupations pédagogiques}

On a souvent accusé les bibliothécaires d'être des gens tranquilles, bien à l'aise et satisfaits à l'intérieur des murs insonorisés de leur bibliothèque, pas très préoccupés des problèmes qui se posent à l'extérieur de ces murs.

Nous pensons que ce jugement, généralisé, est sévère et qu'il faudrait le nuancer très fortement. Dans le milieu collégial, nous avons déjà parlé des échanges d'ordre pédagogique entre des directeurs des études, des professeurs et des bibliothécaires, au Lac Trèfle. Nous pourrions aussi dresser une liste imposante de bibliothécaires de collège qui se sont impliqués au niveau des actions pédagogiques.

\section{Collège-bibliothèque}

II n'est peut-être pas inutile de rappeler 
que ce sont les bibliothécaires qui ont amorcé le déclenchement d'une réflexion du milieu collégial sur cette conception pédagogique.

$\mathrm{Au}$ congrès du Drexel Institute of Technology, tenu à Philadelphie du 18 au 21 décembre 1966, la FCC avait délégué l'auteur de ces lignes pour représenter ses 97 collèges. Le congrès avait pour thème: "The Library College». Deux autres directeurs de bibliothèque de collège participèrent à ces délibérations ainsi que deux bibliothécaires de l'Université de Montréal. Au retour, la conférence de Louis Shores a tout d'abord été publiée en anglais dans la revue Prospectives (3 (1967), 180-190). Une présentation de Victor Coulombe précédait le texte de Louis Shores. Peu de mois après, la version française de cette conférence ainsi que celle de $\mathrm{Sr}$ Helen Sheehan étaient diffusées dans les collèges. Quatre articles portant sur le collègebibliothèque ont été publiés par les bibliothécaires. Une journée d'étude a aussi été consacrée à ce même sujet. Puis, il y a eu l'étude sur le collège-bibliothèque poursuivie au Collège Édouard-Montpetit durant l'année 1968-1969, à laquelle ont été associés un architecte, un pédagogue, un spécialiste en audiovisuel et quatre bibliothécaires. Des bibliothécaires ont aussi été associés à la création du Collège Montmorency qui se voulait une expérience globale du collège-bibliothèque.

L'idée du collège-bibliothèque a été mise en application partiellement dans plusieurs collèges du Québec dont quelquesuns ont délégué des professeurs auprès de collèges américains où la pédagogie du collège-bibliothèque était implantée. Nous pensons que les bibliothécaires ont fourni, dans ce dossier, une contribution qui peut difficilement être niée.

\section{Sessions d'étude portant exclusivement sur des sujets d'ordre pédagogique}

À la Section des collèges comme à la Commission des directeurs de bibliothèque de collège, il y a eu, au cours des années, de nombreuses sessions dont le thème portait exclusivement sur les aspects péda- gogiques de la bibliothèque. L'espace très limité mis à notre disposition nous empêche malheureusement d'en dresser une liste complète.

\section{Les centres de ressources éducatives}

Dans les cégeps, il y a une tendance très nette qui se dessine vers le regroupement de services comme ceux de la bibliothèque, de l'audiovisuel ainsi que de la recherche et de l'expérimentation. Dans les collèges où un tel regroupement s'effectue, les bibliothécaires doivent travailler en collaboration avec d'autres professionnels. Ce n'est peut-être pas toujours facile, mais plusieurs sont parvenus à des réalisations intéressantes par le biais de la collaboration interdisciplinaire.

\section{Stages de La Pocatière}

De 1964 à 1971, des sessions de formation pratique, destinées au personnel technique des bibliothèques, se sont tenues chaque été au Collège de SainteAnne-de-La-Pocatière. Inaugurés par Raymond Boucher, ces stages se présentaient comme une coopérative d'apprentissage où, dans un milieu qui tentait de recréer les conditions de vie d'une bibliothèque idéale, professeurs et tuteurs travaillaient en équipe, guidaient les travaux des stagiaires et fraternisaient avec eux.

\section{Conclusion}

Nous avons énuméré de façon bien insatisfaisante un certain nombre de réalisations des bibliothèques de collège. Plusieurs autres actions auraient mérité d'être soulignées, comme, par exemple, la formation des bibliotechniciens et leur contribution au bon fonctionnement des bibliothèques.

Le milieu des bibliothèques de collège a connu des heures enthousiasmantes, il a produit des chefs de file et, à une période donnée, il a atteint un certain sommet. En cours de route, tout n'a pas toujours réussi. Ainsi, malgré un désir maintes fois exprimé d'introduire l'informatique documentaire 
dans le réseau collégial, malgré le fait que plusieurs bibliothécaires de collège aient participé à des études, à deux missions en France et à des tournées d'observation, malgré des prises de position très précises à certains moments, le bilan de l'automatisation demeure négatif pour les bibliothèques de collège.

Aujourd'hui, le problème des restrictions budgétaires confronte toutes les bibliothèques. Les collèges ont commencé à se pencher sérieusement sur la gestion par objectif, sur l'évaluation des bibliothèques, sur l'élagage des collections et sur l'établissement d'un dépôt semblable au Center for Research Libraries de Chicago ou à d'autres centres de ce genre.

\section{Sources}

Allard, C. Gabriel, c.s.c. Programme pour la construction d'une bibliothèque au Collège d'enseignement général et professionnel de Maisonneuve. Montréal, Bibliothèque du Collège de Maisonneuve, 1971. $75 \mathrm{p}$.

Projet d'un index coopératif des périodiques de langue française pour nos bibliothèques d'enseignement. Montréal, ACBLF, 1965. 42 p. (Travaux et documents, 1).

Bosa, Réal. Liste sélective d'ouvrages de consultation à l'intention des bibliothèques de collèges. Montréal, F.C.C., 1967. iii, 52 p. (Livres et bibliothèques).

Boucher, Raymond. "Le collège-bibliothèque, une solution cadre", Prospectives, vol. 6, no 5 (novembre 1970), 341-347.

Le travail personnel à la bibliothèque au niveau collégial. Conférence prononcée à une journée d'étude de la Section des bibliothèques de collège de l'ACBLF, le 4 février 1962. Montréal, ACBLF, 1962. $17 \mathrm{p}$.

Demers, Jacques. Gestion des documents audiovisuels dans les bibliothèques des collèges francophones du Québec. Montréal, ASTED, 1977. iv, 307 p. (Publications de l'École de bibliothéconomie, 6).

Le service de l'audio-vidéothèque. Document de travail préparé pour la Commission des directeurs de bibliothèque de cégep. Montréal, CADRE, 1971. $18 \mathrm{p}$.

Denis, Laurent-G. Étude sur la gestion des bibliothèques de six collèges classiques de la Province de Québec. Montréal, ACBLF, 1966. ii, 78 p. (Travaux et documents, 2).
Desrochers, Edmond, s.j. Programme pour une bibliothèque collégiale. Montréal, ACBLF, 1961. iv, $82 \mathrm{p}$.

Discrimination du gouvernement à l'égard des bibliothécaires. Rapport du Comité sur le statut et le traitement salarial des bibliothécaires de cégeps. Montréal, CBQ-ASTED, 1974. 85 p.

Fédération des cégeps. Comité conjoint coordonnateurs des techniques audiovisuelles et directeurs de bibliothèque. Audiovidéothèque ou Centre intégré de ressources didactiques? Textes de référence. Montréal, 1972. $123 \mathrm{p}$.

Fédération des cégeps. Commission des directeurs de bibliothèque et Association pour l'avancement des sciences et des techniques de la documentation (ASTED), Section des bibliothèques de collège. Mémoire soumis au Corıseil supérieur de l'Éducation pour son étude sur l'état et les besoins de l'enseignement collégial. Montréal, 1974. 38 p.

Fédération des cégeps. Commission des coordonnateurs de bibliothèque. Normes des bibliothèques de CEGEP. Montréal, 1974. 24 p.

Les normes de bibliothèques de CEGEP. Doc. no 2: quantification. Montréal, 1975. $31 \mathrm{p}$.

Gutzwiller, Alois. Guide du choix des livres en philosophie pour bibliothèques de collèges. Montréal, FCC, 1966. xii, 372 p. (Livres et bibliothèques).

Léveillé, Jean-Bernard. Les bibliothèques de cégeps face au regroupement des ressources éducatives. Montréal, Ministère de l'Éducation, Service des bibliothèques d'enseignement (S.G.M.E.), 1976. $28 \mathrm{p}$.

Les collèges publics du Québec: bilan sur l'aménagement des bibliothèques. Montréal, Ministère de l'Éducation, Service des bibliothèques d'enseignement (S.G.M.E.), 1975. 13 p.

Programme pour la construction d'une bibliothèque au Collège d'enseignement général et professionnel de Saint-Laurent. Montréal, ACBLF, 1971. 78 p. (Travaux et documents, 4).

Livre, bibliothèque et culture québécoise. Mélanges offerts à Edmond Desrochers, s.j. Montréal, ASTED, 1977. $2 \mathrm{v}$.

Ministère de l'Éducation. Service des bibliothèques d'enseignement (S.G.M.E.). Le choix des documents pour les bibliothèques de collège. Rapport du comité «ad hoc». Montréal, 1976. 17 p.

Nadeau, Léonard. Développement des plans et spécifications pour la construction de la bibliothèque du Séminaire Saint-Augustin, Québec. Montréal, F.C.C., 1966. vi, 136 p. (Livres et bibliothèques).

Sansfaçon, Jacques. Liste sélective de périodiques à l'intention des bibliothèques de collèges du Canada français. Trad. d'André Contant. Montréal, F.C.C., 1967. vii, 126 p. (Livres et bibliothèques). 